\title{
Mobility Management for Wireless Systems with Unreliable Backhaul Links
}

\author{
Yi-Bing Lin, Senior Member, IEEE, Shu-Chin Su Chen, and Chu-Sing Yang
}

\begin{abstract}
We consider a wireless system with unreliable microwave backhaul links. Since the links may be disconnected, location update procedure may be incorrectly exercised, which results in misrouted calls. This letter proposes an analytic model to derive the misrouting probabilities as functions of the link disconnection time distribution. These equations can be used as the design guideline to select the microwave equipment for the wireless system.
\end{abstract}

Index Terms-Communication link failure, microwave, mobility management, wireless communications.

\section{INTRODUCTION}

$\mathbf{I}$ N MOBILE SYSTEMS, such as AMPS [1], [2] and GSM [3], interworking between the mobile systems and the PSTN is required for mobility management [4]-[6]. In some areas, interworking functions are not available from the PSTN, and the mobility related messages are delivered within the switches of the wireless system connected by the dedicated links without involvement of the PSTN. An example of such an architecture has been deployed in Mainland China by TECOM. In this system, the mobile switches are connected through microwave backhaul links that are not $100 \%$ reliable (the transmission path may be disconnected due to bad weather or other reasons).

When the microwave links are disconnected, the location update procedure may be affected. In this letter, we propose a location update scheme for wireless system with unreliable backhauls, and utilize an analytic model to study how the reliability of the backhaul links affect the number of misrouted calls. The results can be used as the design guidelines in selecting the microwave equipment for the backhaul connection of the wireless system.

\section{SYSTEM ARCHITECTURE}

Fig. 1 illustrates the architecture of the wireless system with microwave backhaul connections. In this system a local switch (LS) may connect to several base stations. A main switch (MS) is connected to several LS's with microwave links with a star configuration. The links are used for signaling and voice/data call connection. Both the LS's and the MS are connected to PSTN independently through digital trunks (e.g., E1 trunks). Every LS has a visitor location register (VLR)

Manuscript received May 6, 1997. The associate editor coordinating the review of this letter and approving it for publication was Prof. A. K. Elhakeem. This work was supported in part by the National Science Council, Taiwan, R.O.C., under Contract NSC-87-2213-E-009-013.

Y.-B. Lin is with the Department of Computer Science and Information Engineering, National Chiao Tung University, Hsinchu, Taiwan, R.O.C. (email: liny@csie.nctu.edu.tw).

S.-C. S. Chen and C.-S. Yang are with the Institute of Information Engineering, National Sun Yat-Sen University, Taiwan, R.O.C.

Publisher Item Identifier S 1089-7798(98)04026-5.

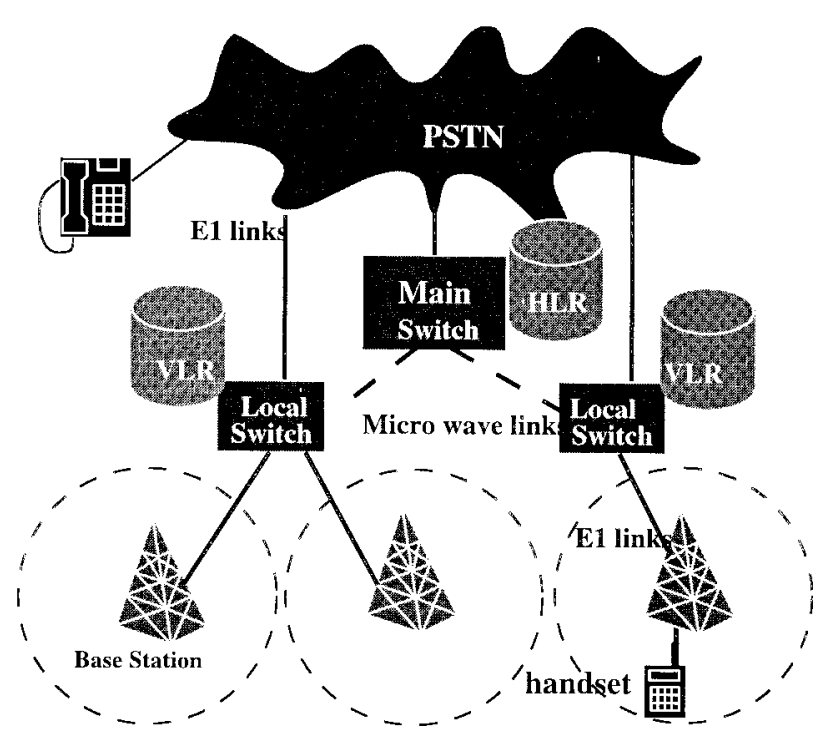

Fig. 1. The wireless system architecture.

to record the mobile users visiting the site. The MS has a home location register (HLR) to record all mobile users in the system. The microwave links between the MS and the LS's may temporarily fail.

This architecture is similar to GSM and AMPS that utilize the concept of HLR/VLR for mobility management. The major difference is that in our system, the signaling protocol for mobility management is proprietary, and signaling does not involve PSTN (i.e., all mobility messages are delivered through the microwave links). The registration procedure (to inform the system the location of the handset) is illustrated in Fig. 2(a). When the handset $p$ moves from area A to area B, the handset registers to the new VLR [in area B; see (1) in Fig. 2(a)]. The new VLR sends a location update message to the HLR, and $p$ 's HLR record indicates that $p$ is in area B [see (2) in Fig. 2(a)]. The HLR sends a cancellation message to the old VLR (in area A) to delete $p$ 's record in the old VLR [see (3) in Fig. 2(a)].

Call termination may occur in one of the three cases. Suppose that the called party (handset $p$ ) is in area B.

Case 1: The caller may be a handset [see (1a) and (2a) in Fig. 2(b)] or a telephone set [see (11b) and (2b) in Fig. 2(b)] connected to the LS in A. The VLR in A is checked, and no record for $p$ is found. The call is forwarded to the MS [see (3) in Fig. 2(b)]. The HLR is queried to find the location of $p$ (which is area B). The call is then forwarded to LS B [see (4) in Fig. 2(b)], and then connected to $p$ [see (5) in Fig. 2(b)].

Case 2: The caller connects to the MS [see (6) in Fig. 2(b)]. The HLR is queried, and the call is set up as in Case 1 [see links (4) and (3) in Fig. 2(b)]. 


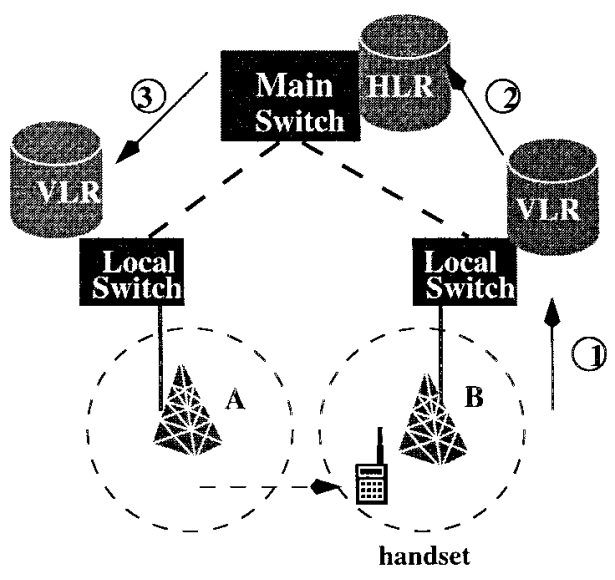

(a)

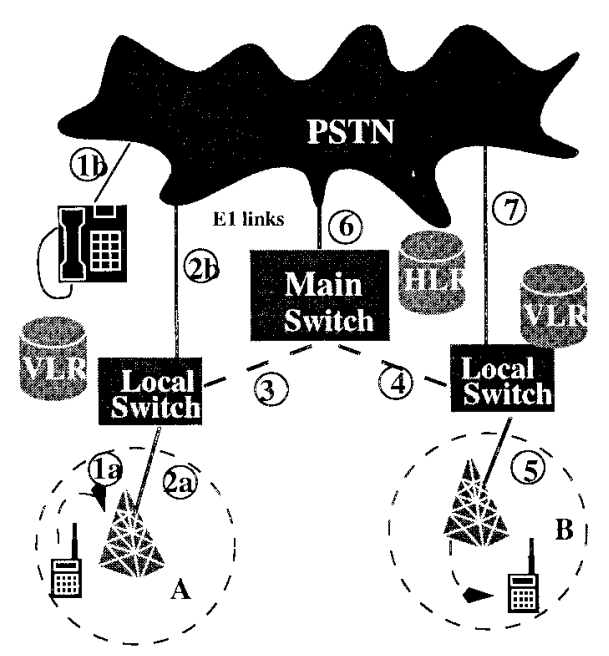

(b)

Fig. 2. Registration and call termination.

Case 3: The caller connects to LS B [see (7) in Fig. 2(b)]. The VLR of B is queried, and the record of $p$ is found. The call is forwarded to $p$ [see (5) in Fig. 2(b)].

\section{Mobility Management with UNRELIABLE BACKHAUL LINKS}

The disconnection of the links affects the registration and the cancellation operations, and the VLR's and the HLR may contain obsolete information. Suppose that the microwave link between the MS and LS B is disconnected when handset $p$ moves from area A to area B in Fig. 2(a). The registration message cannot be forwarded to the MS, and the HLR and the VLR of A have the obsolete location information for $p$ (which indicates that $p$ is in area A). For call terminations in Cases 1 and 2 in Fig. 2(b), the calls are misrouted to the wrong LS.

The database obsoleteness should be limited as transient phenomenon during link disconnection. After link recovery, actions should be taken to guarantee that the information in the databases is current. To achieve this goal, a location update scheme using timestamped signaling messages is proposed. Consider the link between the MS and a local switch LS. During link disconnection, every registration operation from the LS to the MS is marked incomplete. The registration message is sent to the MS as soon as the link is up again. The MS utilizes the timestamp to determine if the registration operation should be performed. The algorithm is described below.

Registration at $L S$ : Suppose that a handset $p$ sends a registration message to LS at time $t_{1}$. LS stores the registration information in its VLR. A record is of the format $\operatorname{rec}_{\mathrm{LS}}[p]=$ (ts, flag, user_profile) where $p$ represents the handset that requests registration, $t s$ represents the time when the registration occurs, flag is 1 (0) if the link between MS and LS is up (down) at time ts, and user_profile provides necessary information (such as call restrictions, call-waiting allowed, call-forwarding allowed, and so on) to set up calls for the mobile user. If the link to MS is up, then a registration message $m s g=\left(\mathrm{LS}, p, t_{1}\right)$ is sent to MS and $\operatorname{rec}_{\mathrm{LS}}[p] \cdot f l a g=1$. Otherwise, $\operatorname{rec}_{\mathrm{LS}}[p]$.flag $=0$.

Link Recovery at LS: Suppose that the disconnected link between MS and LS becomes up at time $t_{2}$. For every location record $r c_{\mathrm{LS}}[p]=\left(t_{1}, 0\right.$, user_profile $)$, the flag field is set to 1 , and a registration message $m s g_{r}=\left(\mathrm{LS}, p, t_{1}\right)$ is sent to MS (note that $t_{1}<t_{2}$ ).

Registration at MS: A location record in MS is of the format rec $_{\mathrm{MS}}[p]=(l s, t s$, user_profile $)$ where $p$ is the handset, $l s$ indicates the local switch where the handset resides, and $t s$ indicates the time of last registration. When MS receives a registration message $m s g_{r}=\left(\mathrm{LS}, p, t_{1}\right)$, it checks if $t_{1}>$ $r c_{\mathrm{MS}}[p] . t s$. If so, the registration is effective, and $\mathrm{LS}_{\text {old }}=$ $\operatorname{rec}_{\mathrm{MS}}[p] . \mathrm{LS}$ ( $\mathrm{LS}_{\mathrm{old}}$ will be used in the cancellation operation), $\operatorname{rec}_{\mathrm{MS}}[p] . l s=\mathrm{LS}$, and $\operatorname{rec}_{\mathrm{MS}}[p] . t s=t_{1}$. Otherwise, the registration is ineffective, and the registration message is ignored.

Cancellation at MS: After an effective registration at $\operatorname{rec}_{\mathrm{MS}}[p]$ is completed, MS attempts to send a cancellation message $m s g_{c}=(p, \operatorname{rec}[p] . t s)$ to the old local switch $\mathrm{LS}_{\text {old }}$. If the microwave link between MS and $\mathrm{LS}_{\text {old }}$ is up, then $m s g$ is delivered. Otherwise, $m s g$ is buffered in a queue $Q\left[\mathrm{LS}_{\text {old }}\right]$.

Link Recovery at MS: Suppose that the microwave link between MS and LS is up. Then MS sends all cancellation messages in $Q[\mathrm{LS}]$ to LS.

Cancellation at LS: Suppose that LS receives a cancellation message $m s g_{c}=\left(p, t_{1}\right)$ from MS. If $\operatorname{rec}_{\mathrm{LS}}[p] . t s<t_{1}$, then the cancellation is effective and $\operatorname{rec}_{\mathrm{LS}}[p]$ is removed. Otherwise, the cancellation is ineffective and is ignored.

\section{Performance Analysis}

A call may be misrouted when the microwave links are disconnected. The number of misrouted calls is determined by the down times of the links, the call arrival rate, and the mobility of the users. Since the cost of maintaining highly reliable microwave links is expensive, the network designer may consider a less expensive (and thus less reliable) microwave equipment if the number of misrouted calls is sufficiently small. Performance modeling of the system is required to provide the design guidelines. Specifically, we would like to know how the number of misrouted calls are affected by changing the disconnection times of the microwave links.

An analytic model is described as follows. Consider the timing diagram in Fig. 3(a). Suppose that the microwave link between a LS and the MS is disconnected during $\left[t_{0}, t_{2}\right]$. Suppose that the disconnection period $T=t_{2}-t_{0}$ is a random 


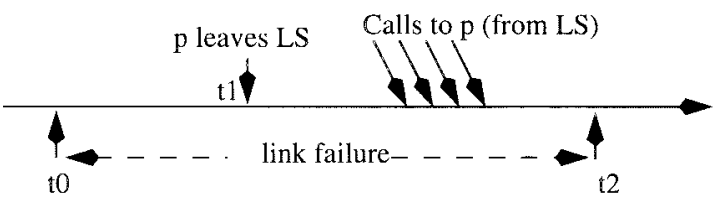

(a)

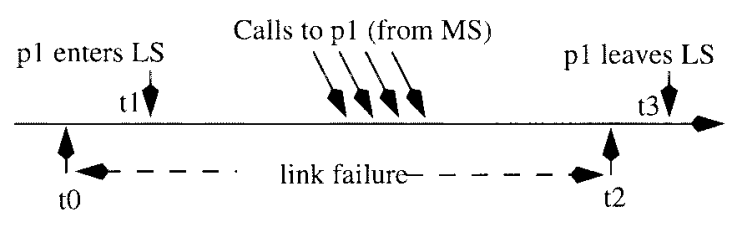

(b)

Fig. 3. The timing diagram.

variable with the density function $f(t)$ with mean $1 / \mu$. A handset $p$ leaves LS at time $t_{1}$. Since the link between LS and MS is disconnected, cancellation operation [(3) in Fig. 2(a)] cannot be performed. Thus all calls to $p$ [the slant arrows in Fig. 3(a)] directly from PSTN to LS (i.e., Case 1 in Fig. 2(b)] are misrouted during $\left[t_{1}, t_{2}\right]$. These calls are called Type $I$ misrouted calls. Suppose that the calls (for $p$ ) directly from PSTN to LS are Poisson arrivals with rate $\lambda_{1}$. If the leaving of $p$ is a random observer [7] of the period $T$, then from the residual life theorem, $t=t_{2}-t_{1}$ is a random variable with the distribution function $R(t)$ and the density function $r(t)=\mu \int_{\tau=t}^{\infty} f(\tau) d \tau$. Let $\operatorname{Pr}_{I}[K=k]$ be the probability that there are $k$ Type I misrouted calls for $p$ during the period $T$. Then

$$
\begin{aligned}
\operatorname{Pr}_{I}[K=k] & =\int_{t=0}^{\infty} \frac{\left(\lambda_{1} t\right)^{k}}{k !} e^{-\lambda_{1} t} r(t) d t \\
& =\left.\left[\frac{\left(-\lambda_{1}\right)^{k}}{k !}\right]\left[\frac{d^{k} r^{*}(s)}{d^{k} s}\right]\right|_{s=\lambda_{1}}
\end{aligned}
$$

where $r^{*}(s)$ is the Laplace Transform for $R(t)$. Considered the timing diagram in Fig. 3(b). Suppose that a handset $p_{1}$ enters LS at time $t_{1}$ and leaves LS at time $t_{3}$. Let the LS residence time $t^{\prime}=t_{3}-t_{1}$ be a random variable with the distribution function $H\left(t^{\prime}\right)$, the density function $h\left(t^{\prime}\right)$, the Laplace transform $h^{*}(s)$, and mean $1 / \eta$. Then all calls to $p_{1}$ [the slant arrows in Fig. 3(b)] from MS (Cases 1 and 2 in Fig. 2(b)] during the period $\left[t_{1}, \min \left(t_{2}, t_{3}\right)\right]$ are misrouted (referred to as the Type II misrouting), and the period $x=$ $\min \left(t_{2}, t_{3}\right)-t_{1}$ has the density function

$$
\begin{aligned}
r_{1}(x) & =\int_{\tau=x}^{\infty}[r(x) h(\tau)+r(\tau) h(x)] d \tau \\
& =r(x)+h(x)-r(x) H(x)-h(x) R(x)
\end{aligned}
$$

and the Laplace transform

$$
r_{1}^{*}(s)=r^{*}(s)+h^{*}(s)-f_{r H}^{*}(s)-f_{h R}^{*}(s)
$$

where (from [8])

$$
f_{r H}^{*}(s)=\frac{1}{2 \pi j} \int_{\sigma=c-j \infty}^{c+j \infty}\left[\frac{r^{*}(\sigma) h^{*}(s-\sigma)}{s-\sigma}\right] d \sigma
$$

$$
f_{h R}^{*}(s)=\frac{1}{2 \pi j} \int_{\sigma=c-j \infty}^{c+j \infty}\left[\frac{h^{*}(\sigma) r^{*}(s-\sigma)}{s}\right] d \sigma .
$$

Let $\operatorname{Pr}_{I I}[K=k]$ be the probability that there are $k$ Type II misrouted calls for $p_{1}$ during the period $x$. Assume that the calls to $p_{1}$ through MS are Poisson arrivals with rate $\lambda_{2}$. Then similar to (1),

$$
\begin{aligned}
\operatorname{Pr}_{I I}[K=k] & =\int_{x=0}^{\infty} \frac{\left(\lambda_{2} x\right)^{k}}{k !} e^{-\lambda_{2} x} r_{1}(x) d x \\
& =\left.\left[\frac{\left(-\lambda_{1}\right)^{k}}{k !}\right]\left[\frac{d^{k} r_{1}^{*}(s)}{d^{k} s}\right]\right|_{s=\lambda_{1}} .
\end{aligned}
$$

Note that both (1) and (4) are general solutions that accommodate arbitrary link failure density function $f(t)$ and LS residence time density function $h(t)$. To approximate the effects of the means of the link failure times and the LS residence times, let both $f(t)$ and $h(t)$ be exponential. Thus, (1) and (4) are rewritten as

$$
\begin{aligned}
& \operatorname{Pr}_{I}[K=k]=\frac{\mu \lambda_{1}^{k}}{\left(\lambda_{1}+\mu\right)^{k+1}} \\
& \operatorname{Pr}_{I I}[K=k]=\frac{(\eta+\mu) \lambda_{2}^{k}}{\left(\eta+\mu+\lambda_{2}\right)^{k+1}} .
\end{aligned}
$$

Equation (5) can be used as the guideline to determine how reliable the microwave links should be. For example, if the system is designed such that the probability of any misrouted call is limited to be less than $\alpha$ (where $0<\alpha<1$ ), then the mean microwave link down time $1 / \mu$ should satisfy the constraint that

$$
\mu>\max \left[\frac{\alpha\left(\eta+\lambda_{2}\right)-\eta}{1-\alpha}, \frac{\lambda_{1}}{1-\alpha}\right] .
$$

\section{SUMMARY}

This letter studied the effect of the unreliable microwave backhaul links on the number of misrouted calls in a wireless system. When the backhaul links are down, the location update procedure can not be correctly exercised, and some calls may be misrouted. An analytic model is developed to study how the microwave link down time distribution affects the misrouted calls. The derived misrouted probabilities can be used as the guidelines to select the microwave equipment.

\section{REFERENCES}

[1] ANSI/EIA/TIA, "Mobile station-land station compatibility specification," Tech. Rep. 553, EIA/TIA, 1989.

[2] W. C. Y. Lee, Mobile Cellular Telecommunications Systems. New York: McGraw-Hill, 1995.

[3] S. Redl and M. Weber, An Introduction to GSM. Reading MA: Artech House, 1995.

[4] ETSI/TC, "Mobile application part (MAP) specification, version 4.8," Tech. Rep. Recommendation GSM 09.02, ETSI, 1994.

[5] Y.-B. Lin and S. K. DeVries, "PCS network signaling using SS7," IEEE Personal Commun. Mag., pp. 44-55, June 1995.

[6] EIA/TIA, "Cellular intersystem operations (rev. C)," Tech. Rep. IS-41, EIA/TIA, 1995.

[7] P. J. B. King, Computer and Communication Systems Performance Modeling. Englewood Cliffs, NJ: Prentice Hall, 1990.

[8] E. J. Watson, Laplace Transforms and Applications. Birkhauser, 1981. 